\title{
Multi-Source Multi-Sensor Information Fusion
}

\section{FOREWORD}

The human brain routinely carries out information processing and fusion. The objective is to collect observations from various similar or dissimilar sources and sensors, extract the required information (inferences) and combine/fuse these with a view to obtaining an enhanced status and identity of a perceived object. This process that is very crucial for survival and growth of human beings (as well as most other living creatures) can be termed as multi-source multi-sensor information fusion (MUSSIF).

MUSSIF is rapidly emerging as a discipline to reckon with and is finding ever-increasing applications in biomedical, industrial automation, aerospace systems and environmental engineering. This is expected to give better spatial coverage, redundancy, robustness and accuracy. The complete process of MUSSIF can involve study of several allied disciplines: signal/image processing, numerical algorithms, statistical estimation, sensor management, control and optimization, neural networks, fuzzy systems, and data base management. Many principles and techniques from these fields carry over to the process of MUSSIF.

This special issue is brought out with the aim of consolidating some recent efforts in the area of MUSSIF. There are six papers, which elucidate data fusion strategies and algorithms, performance evaluation and achievable accuracy, mainly for aerospace applications.

The paper by Subhash Challa and Don Koks gives a good review of the recent data fusion literature and clearly elucidates the concepts of Bayesian and Dempster-Shafer fusion methods. A new derivation of the Kalman Filter is given. The authors illustrate several important aspects of these two approaches by taking examples of fusion of data from two sensors.

The paper by B N Suresh and K Sivan deals with types of sensors used in launch vehicles and their characteristics. The authors describe performance evaluation aspects. The paper dwells on the integrated NGC-PC (navigation, guidance, and control-propulsion control) system and sensors, sensor data processing and performance requirement, sensor redundancy management, and performance evaluation of the NGC-PC system. The authors stress the importance of multi-sensor systems with proper redundancy to achieve the goal of accurately injecting a satellite into a pre-defined orbit.

The paper by $\mathrm{N}$ Venkateswaran et al deals with fusion of data from gyro and horizon sensors for precision pointing of imaging spacecraft. Brief descriptions of these sensors, data processing, and fusion aspects are dealt with. A Kalman Filter is used for fusion of sensor data and performance of the scheme is evaluated.

The paper by V Vaidehi, S Indira Gandhi and K Kalavidya discusses the importance of interactive multiple modelling approach for tracking of maneuvering targets and its implementation using a cluster of processors in a centralized architecture of data fusion. The cluster used by the authors has given $88 \%$ efficiency and speedup of 3.52 .

$\mathrm{N}$ Shanthakumar and $\mathrm{T}$ Jann describe an important application of fusion of information from a low-cost inertial platform system using Kalman filters. They use MATLAB/SIMULINK for simulation validation of the fusion scheme and give results of the implementation of the Kalman Filter-based scheme on a micro controller. 
R Appavu Raj and Shrabani Bhattacharya give results of performance evaluation of multisensor data fusion applications to target tracking with simulation and real data.

All the authors must be complimented for their valuable contributions to this special issue. The referees have done a good job of reviewing these papers. Thanks are due to Dr. Gangan Prathap, Editor of $S \bar{a} d h a n \bar{a}$, for the invitation to compile the issue. Also thanks are due to Ms. K Shashikala \& Mr Riki Krishnan of Sädhanā for their very cordial support during the process.

April 2004

JITENDRA R RAOL

Guest Editor

Flight Mechanics \& Control Division

National Aerospace Laboratories

P.B. No. 1779, Bangalore 560017

India 